The first mock data challenge for LISA Pathfinder

This article has been downloaded from IOPscience. Please scroll down to see the full text article.

2009 Class. Quantum Grav. 26094004

(http://iopscience.iop.org/0264-9381/26/9/094004)

View the table of contents for this issue, or go to the journal homepage for more

Download details:

IP Address: 194.94.224.254

The article was downloaded on 08/10/2010 at 09:34

Please note that terms and conditions apply. 


\title{
The first mock data challenge for LISA Pathfinder
}

\author{
A Monsky ${ }^{1}$, M Hewitson ${ }^{1}$, L Ferraioli ${ }^{2}$, G Wanner ${ }^{1}$, M Nofrarias ${ }^{1}$, \\ M Hueller $^{2}$, I Diepholz ${ }^{1}$, A Grynagier ${ }^{3}$, M Armano $^{4}$, M Benedetti $^{5}$, \\ J Bogenstahl $^{6}$, D Bortoluzzi ${ }^{7}$, P Bosetti ${ }^{7}$, N Brandt ${ }^{8}$, A Cavalleri ${ }^{2}$, \\ G Ciani $^{2}$, I Cristofolini ${ }^{7}$, M Cruise $^{9}$, K Danzmann $^{1}$, R Dolesi $^{2}$, J Fauste $^{4}$, \\ D Fertin $^{10}$, W Fichter $^{3}$, A García ${ }^{1}$, C García ${ }^{10}$, F Guzmán ${ }^{1}$,

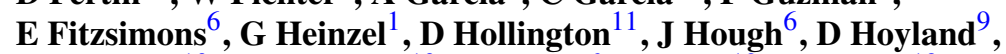 \\ O Jennrich $^{10}$, B Johlander ${ }^{10}$, C Killow ${ }^{6}$, A Lobo ${ }^{12}$, D Mance ${ }^{13}$, \\ I Mateos $^{12}$, P W McNamara ${ }^{10}$, D Nicolini ${ }^{10}$, D Nicolodi ${ }^{2}$, \\ M Perreur-Lloyd $^{6}$, E Plagnol $^{14}$, G D Racca ${ }^{10}$, J Ramos-Castro $^{15}$, \\ D Robertson $^{6}$, J Sanjuan ${ }^{12}$, M O Schulte ${ }^{11}$, D N A Shaul ${ }^{11}$, M Smit ${ }^{16}$, \\ L Stagnaro ${ }^{10}$, F Steier ${ }^{1}$, T J Sumner ${ }^{11}$, N Tateo ${ }^{2}$, D Tombolato ${ }^{2}$,

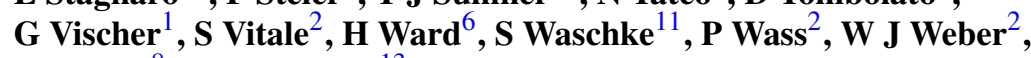 \\ T Ziegler ${ }^{8}$ and $\mathbf{P}$ Zweifel $^{13}$
}

1 Albert-Einstein-Institut, Max-Planck-Institut für Gravitationsphysik und Universität Hannover, 30167 Hannover, Germany

2 Dipartimento di Fisica, Università di Trento and INFN, Gruppo Collegato di Trento, 38050 Povo, Trento, Italy

3 Institut für Flugmechanik und Flugregelung, 70569 Stuttgart, Germany

${ }^{4}$ European Space Agency, ESAC, Villanueva de la Cañada, 28692 Madrid, Spain

5 Dipartimento di Ingegneria dei Materiali e Tecnologie Industriali,

Università di Trento and INFN, Gruppo Collegato di Trento, Mesiano, Trento, Italy

${ }^{6}$ Department of Physics and Astronomy, University of Glasgow, Glasgow, UK

${ }^{7}$ Dipartimento di Ingegneria Meccanica e Strutturale, Università di Trento and INFN,

Gruppo Collegato di Trento, Mesiano, Trento, Italy

${ }^{8}$ Astrium GmbH, 88039 Friedrichshafen, Germany

${ }^{9}$ Department of Physics and Astronomy, University of Birmingham, Birmingham, UK

${ }^{10}$ European Space Agency, ESTEC, 2200 AG Noordwijk, The Netherlands

11 The Blackett Laboratory, Imperial College, London, UK

12 Institut d'Estudis Espacials de Catalunya and Institut de Ciencies de 1'Espai, CSIC, 08034 Barcelona, Spain

${ }^{13}$ Institut für Geophysik, Eidgenössische Technische Hochschule Zürich, CH-8093, Zürich, Switzerland

14 APC UMR7164, Université Paris Diderot, Paris, France

15 Universitat Politècnica de Catalunya, 08034 Barcelona, Spain

16 SRON Netherlands Institute for Space Research, 3584 CA Utrecht, The Netherlands

E-mail: anneke.monsky@aei.mpg.de

Received 31 October 2008, in final form 19 February 2009

Published 20 April 2009

Online at stacks.iop.org/CQG/26/094004

\begin{abstract}
The data analysis of the LISA Technology Package (LTP) will comprise a series of discrete experiments, each focusing on a particular noise measurement or characterization of the instrument in various operating modes. Each of these
\end{abstract}


experiments must be analysed and planned in advance of the mission because the results of a given experiment will have an impact on those that follow. As such, a series of mock data challenges (MDCs) will be developed and carried out with the aim of preparing the analysis tools and optimizing the various planned analyses. The first of these MDCs (MDC1) is a simplified treatment of the dynamics along the axis joining the two test masses onboard LISA Pathfinder. The validation of the dynamical model by predicting the spectra of the interferometer output data is shown, a prediction for the data analysis is calculated and, finally, several simulated interferometer data sets are analysed and calibrated to equivalent out-of-loop test mass acceleration.

PACS numbers: $95.55 . \mathrm{Ym}, 04.80 . \mathrm{Nn}$

(Some figures in this article are in colour only in the electronic version)

\section{Introduction}

LISA Pathfinder is an ESA space mission designed to test critical technologies for the joint ESA/NASA mission LISA. The main mission goal of the LISA Technology Package (LTP) aboard LISA Pathfinder is the verification of free-fall between two test masses (TM) with an accuracy of about $3 \times 10^{-14} \mathrm{~ms}^{-2} \mathrm{~Hz}^{-1 / 2}\left[1+(\mathrm{f} / 3 \mathrm{mHz})^{2}\right]$ in a measurement bandwidth (MBW) between $1 \mathrm{mHz}$ and $30 \mathrm{mHz}$ [1]. For the data analysis of LTP a series of mock data challenges (MDC) will be developed. The first of these MDCs (MDC1) is the subject of this paper. It deals with the dynamics of the test masses aboard LISA Pathfinder. The optical metrology system (OMS) of LTP comprises two measurement interferometers: the so-called $\mathrm{X} 1$ interferometer measures the distance between test mass 1 and the spacecraft (indicated as $x_{1}$ in figure 1), and the X12 interferometer gives the distance between both test masses. Further details about the OMS can be found in [2].

The main task of the first mock data challenge and so of this paper is the calibration of simulated interferometer data, the so-called mock data, to test mass acceleration. For this purpose, a couple of time series containing X1 and X12 mock interferometer data as well as a set of agreed spectra of noise sources were provided by a sub-group of the data analysis group from the University of Trento. The procedure of this data generation is documented in [3] and shall not be considered in this paper. Instead, the paper focuses on the calibration of the simulated data to test mass acceleration and on the verification of the model that has been used to simulate the data. The work presented here can be seen mostly independent from the data generation. Also the software as well as the analysing tools was different from those used for the generation of the mock data.

The model underlying MDC1 is a simplified model of the LTP dynamics. Section 2 deals with the set-up of this model and its mathematical description. This mathematical description of the dynamics of LTP is essential for the realization of MDC1. The procedure to calibrate the received mock interferometer data to test mass acceleration divides into three main parts:

Predicting the spectral content of the mock data (see section 3). Tests the mathematical interpretation of the model derived for the data analysis in section 2 . This is done by substituting all given information provided by the data generation team namely the analytical functions of all noise sources into the derived dynamical model to obtain an analytical expression for the 
$\mathrm{A}_{\mathrm{df}} \quad\left[\frac{\mathrm{N}}{\mathrm{kg}}\right] \quad$ - thruster force per unit mass

$\mathrm{A}_{\mathrm{N}} \quad\left[\frac{\mathrm{N}}{\mathrm{kg}}\right]$ - acceleration noise on spacecraft

$k_{1} x_{1} \quad[\mathrm{~N}] \quad$ - spring coupling between TM1 and S/C

$k_{3} x_{2} \quad[\mathrm{~N}] \quad$ - spring coupling between TM2 and S/C

$\mathrm{A}_{1}, \mathrm{~A}_{2}\left[\frac{\mathrm{N}}{\mathrm{kg}}\right]$ - acceleration noises on TM1 and TM2

$\mathrm{C}_{\mathrm{df}} \quad\left[\mathrm{s}^{-2}\right]$ - drag-free controller

$\mathrm{C}_{\text {sus }} \quad\left[\mathrm{s}^{-2}\right]$ - electrostatic suspension controller

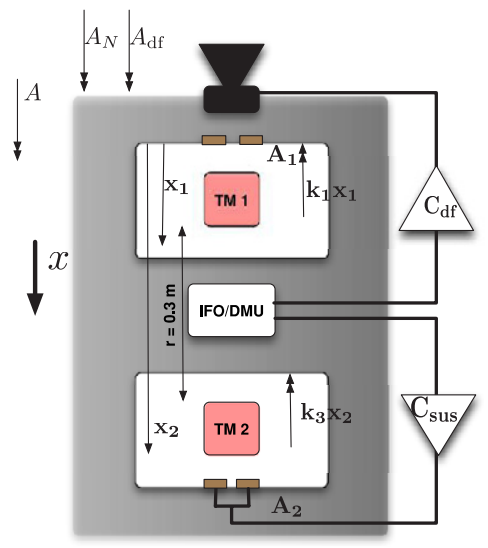

Figure 1. Simplified model of the LTP dynamics. The arrows of $A$ and $x$ indicate the positive direction of forces (per unit mass) and distances reflected in equations (4)-(6). $x_{1}$ and $x_{2}$ are the distances between the spacecraft and TM1 and TM2, respectively. The block IFO/DMU represents the interferometer and the data management unit. More detailed information on the DMU can be found in [2].

$\mathrm{X} 1$ and X12 interferometer data. If the model derived in section 2 of this paper is correct, the resulting spectra will match the spectra of the mock data and the model is verified. If these two spectra happen to be different, this would indicate that the model used for the simulation of the data differs from that derived here.

Predicting the test mass accelerations (see section 3). Gives a reference for the analysis of the simulated data (see the next step). By applying the analytical functions of the noise sources acting on LTP to the dynamical model verified in the previous step, it is also possible to compute an analytical function for test mass acceleration. The result will be a frequency-dependent function representing the test mass acceleration resulting from the applied forces.

Calibrating the mock data to test mass acceleration (see section 3). This is the main goal of MDC1. In this step, the verified dynamical model of LTP is used to calibrate mock interferometer data to test mass acceleration just like it could be done using real mission data, only with a much more complicated model. This is the mission like data analysis, which means that we will not make use of any noise sources, because they will be unknown during the mission. Instead, the data are analysed by applying our knowledge about the dynamical behaviour of the system and eventually parameters and forces that are measurable. Since the simulated interferometer data are time series just like the data being downlinked from the satellite will be, the analysis can be carried out either in the frequency or in the time domain. Either way, the resulting spectrum of the test mass accelerations can now be compared to the prediction of these accelerations computed earlier.

The complete analysis has been carried out using LTPDA, a comprehensive analysis tool based on MATLAB, especially developed for the data analysis of LTP. It is used (and thereby tested extensively) during the development phase for data analysis of ongoing experiments mostly at the Albert Einstein Institute and the Physics department of the University of Trento, but also at other institutions. Detailed information on this can be found in [4]. 


\section{The model}

The model underlying MDC1 describes a simplified set-up of LTP, where the two test masses move only along the sensitive $x$-axis while no cross-talk from other axes is accounted for. A schematic of this model is shown in figure 1. It shows a simplified model of the spacecraft (S/C) including the two test masses (TM1 and TM2), the capacitive sensors, the microthrusters and the controllers, as well as all forces and interactions acting along the sensitive axis. As indicated in figure 1, the position of TM1 relative to the spacecraft is controlled by the thrusters via the drag-free loop whereas the capacitive actuators control the position of TM2 relative to the spacecraft via the electrostatic suspension loop [5]. The model describes the so-called science mode, where TM1 is kept in free-fall within the MBW, and the spacecraft is controlled such that it follows the motion of TM1. The position of TM2 is controlled such that the distance between the two test masses is kept constant and thereby the position of TM2 with respect to the spacecraft. Analysing all forces acting on the system shown in figure 1 will lead to the equations of motion of the two test masses, and from these equations their accelerations can be computed. One way to find these equations is setting up the Lagrangians and solving the Euler-Lagrange equations of the system. The Lagrangian is defined as the kinetic energy, $T$, of the system minus its potential energy, $V$, which can be written as $L=T-V$. For the simplified model of LTP the Lagrangian reads

$$
L=\frac{m}{2} \dot{x}_{1}^{2}+\frac{m}{2} \dot{x}_{2}^{2}-\frac{k_{1}}{2} x_{1}^{2}-\frac{k_{2}}{2}\left(x_{2}-x_{1}\right)^{2}-\frac{k_{3}}{2} x_{2}^{2} .
$$

Here the term $\frac{k_{2}}{2}\left(x_{2}-x_{1}\right)^{2}$ represents the gravitational coupling between the test masses, with $k_{2}$ being the spring constant. From this, the Euler-Lagrange equations are found for TM1 and TM2, respectively:

$$
\begin{array}{ll}
\text { external force on TM1: } & F_{1}=\frac{\partial L}{\partial x_{1}}-\frac{\mathrm{d}}{\mathrm{d} t}\left(\frac{\partial L}{\partial \dot{x}_{1}}\right), \\
\text { external force on TM2: } & F_{2}=\frac{\partial L}{\partial x_{2}}-\frac{\mathrm{d}}{\mathrm{d} t}\left(\frac{\partial L}{\partial \dot{x}_{2}}\right) .
\end{array}
$$

In an inertial frame, equations (2) and (3) would be equal to zero. The LTP experiment however is a non-inertial frame, where forces acting on the spacecraft are external. This leads to a total force on TM1 and TM2 which is non-zero. In the following, the external forces ( $F_{1}$ and $F_{2}$ ) acting on the system will be derived. The mass of the spacecraft is denoted by $M$, and $m$ is the mass of each test mass. The forces acting on the spacecraft are:

- the force applied by the thrusters: $M A_{\mathrm{df}}$,

- additional force noises like solar radiation pressure and un-commanded thruster noise: $M A_{\mathrm{N}}$,

- forces arising from the back reaction of the spring coupling of the test masses: $-k_{1} x_{1}$ and $-k_{3} x_{2}$.

The total force on the spacecraft can then be written as

$$
F_{\mathrm{SC}}=M A_{\mathrm{sc}}=M A_{\mathrm{df}}+M A_{\mathrm{N}}-k_{1} x_{1}-k_{3} x_{2} .
$$

External forces on TM1 arise from the pseudoforce from the spacecraft, due to working in a non-inertial frame $\left(-m A_{\mathrm{sc}}\right)$. Additional forces $\left(m A_{1}\right)$ may arise from local disturbances, for example, magnetic fields. This leads to the expression for the external force on TM1:

$$
F_{1}=m A_{1}-m A_{\mathrm{sc}}=m A_{1}-m A_{\mathrm{df}}-m A_{\mathrm{N}}+\mu k_{1} x_{1}+\mu k_{3} x_{2},
$$

where $\mu=m / M$. 
A force from the electrostatic actuator $\left(m A_{\text {sus }}\right)$ acts on TM2, which leads to the following expression for the external force on TM2:

$$
F_{2}=m A_{2}+m A_{\text {sus }}-m A_{\mathrm{df}}-m A_{\mathrm{N}}+\mu k_{1} x_{1}+\mu k_{3} x_{2} .
$$

The thruster force, $M A_{\mathrm{df}}$, arises from the drag-free loop reading the output of the $\mathrm{X} 1$ interferometer and feeding back to the micro-Newton thrusters to make the spacecraft follow TM1. Therefore it reads

$$
F_{\mathrm{df}}=M A_{\mathrm{df}}=o_{1} C_{\mathrm{df}} M,
$$

with $C_{\mathrm{df}}$ being the transfer function of the drag-free controller, and $o_{1}$ the $\mathrm{X} 1$ interferometer output.

The electrostatic suspension loop reads out the differential interferometer output, $o_{\Delta}$, and feeds back to TM2. The force arising from this reads

$$
F_{\text {sus }}=m A_{\text {sus }}=o_{\Delta} C_{\text {sus }} m,
$$

where $C_{\text {sus }}$ is the transfer function of the electrostatic suspension controller. Inserting equation (5) into equation (7) and computing (2) and inserting equation (6) into equation (8) and computing (3), yields the final equations of motion for TM1 and TM2, respectively:

$$
\begin{aligned}
& {\left[s^{2}+\omega_{1}^{2}(1+\mu)+\mu \omega_{3}^{2}\right] x_{1}+\left[\mu \omega_{3}^{2}-\omega_{2}^{2}\right] x_{12}=o_{1} C_{\mathrm{df}}+A_{\mathrm{N}}-A_{1},} \\
& {\left[s^{2}+2 \omega_{2}^{2}+\omega_{3}^{2}\right] x_{12}+\left[\omega_{3}^{2}-\omega_{1}^{2}\right] x_{1}=A_{1}-A_{2}-o_{\Delta} C_{\text {sus }},}
\end{aligned}
$$

where the equations have been moved to the Laplace domain, with $s=\mathrm{i} \omega$ acting as the Laplace variable for easier calculation of the derivatives and $\omega_{1}^{2}=\frac{k_{1}}{m}, \omega_{2}^{2}=\frac{k_{2}}{m}, \omega_{3}^{2}=\frac{k_{3}}{m}$ are the so-called stiffness terms.

Equations (9) and (10) can be simplified further, taking into account that $\mu$ is defined as the mass of the test mass divided by the mass of the spacecraft. Therefore, it can be assumed that this ratio is small compared to 1 . Furthermore, the gravitational coupling between the test masses, $\omega_{2}$, is small compared to $\omega_{3}$, the coupling between TM 2 and the spacecraft.

These simplifications are applied in the next step. Moreover, the formalism can be compressed to the following equation when it is converted to matrix representation:

$$
\mathbf{D} \vec{q}=\mathbf{C} \vec{o}+\vec{g}_{\mathrm{n}},
$$

where the following definitions have been used:

$$
\begin{array}{ll}
\vec{q}=\left[\begin{array}{l}
x_{1} \\
x_{\Delta}
\end{array}\right], & \vec{o}=\left[\begin{array}{l}
o_{1} \\
o_{\Delta}
\end{array}\right], \\
\mathbf{D}=\left[\begin{array}{cc}
\left(s^{2}+\omega_{1}^{2}\right) & 0 \\
\omega_{\Delta}^{2} & \left(s^{2}+\omega_{3}^{2}\right)
\end{array}\right]
\end{array}
$$

with $\omega_{\Delta}=\omega_{3}-\omega_{1}, x_{\Delta}=x_{2}-x_{1}$ and $\vec{o}$ being the interferometer output.

Both controller transfer functions include well-defined delays: $\Xi_{\mathrm{df}}$ for the drag-free loop and $\Xi_{\text {sus }}$ for the suspension loop, as well as actuator delays $G_{\mathrm{df}}$ and $G_{\text {sus }}$, respectively. These delays result from the time needed for the data transmission and calculation of the commanded force. Moreover, the actuator does not respond instantaneously. This is modelled by a low-pass filter in both actuators (micro-thruster and electrostatics) [6]. The matrices for the actuation $(\mathbf{G})$, the time delays $(\boldsymbol{\Xi})$ and the controller transfer functions $\left(\mathbf{C}_{\mathrm{tf}}\right)$ are then defined as follows:

$\boldsymbol{\Xi}=\left[\begin{array}{cc}\Xi_{\mathrm{df}} & 0 \\ 0 & \Xi_{\mathrm{sus}}\end{array}\right], \quad \mathbf{G}=\left[\begin{array}{cc}G_{\mathrm{df}} & 0 \\ 0 & G_{\mathrm{sus}}\end{array}\right], \quad \mathbf{C}_{\mathrm{tf}}=\left[\begin{array}{cc}C_{\mathrm{tf}_{1}} & 0 \\ 0 & C_{\mathrm{tf}_{2}}\end{array}\right]$. 
The complete controller matrix reads

$$
\mathbf{C}=\left[\begin{array}{cc}
C_{\mathrm{df}} & 0 \\
0 & C_{\mathrm{sus}}
\end{array}\right]=\mathbf{\Xi} \mathbf{G C}_{\mathrm{tf}} .
$$

Moreover, the interferometer output data and the TM coordinates can be related by the simplified interferometer cross-talk matrix:

$$
\mathbf{S}=\left[\begin{array}{ll}
1 & 0 \\
\delta & 1
\end{array}\right]
$$

The interferometer output can now be expressed as

$$
\vec{o}=\mathbf{S} \vec{q}+\vec{o}_{\mathrm{n}}
$$

with $\vec{o}_{\mathrm{n}}$ being the interferometer sensing noise. From equation (16) it can be seen that the position of TM1 couples into the differential interferometer output, $o_{\Delta}$, by a coupling factor $\delta$. This means that if both test masses are moving but their distance keeps constant, the X12 interferometer still detects a signal $o_{\Delta}$. This coupling factor arises from the interferometer design and possible imperfections in the alignment.

Combining equations (11) and (16) leads to an expression for the acceleration of the test masses that separates the well-known parameters such as the dynamics, the interferometer cross-talk, the controller functions and the interferometer output data (left-hand side of equation (17)) from the unknown ones that are the noise sources (right-hand side of equation (17)). The analysis of the system for MDC1 is fully described in the following equation:

$$
\left[\mathbf{D S}^{-1}-\mathbf{C}\right] \vec{o}=\mathbf{D S}^{-1} \vec{o}_{\mathrm{n}}+\vec{g}_{\mathrm{n}} .
$$

The right-hand side of (17) will be denoted as predicted acceleration:

$$
\vec{a}_{\text {pred }}=\mathbf{D S}^{-1} \vec{o}_{\mathrm{n}}+\vec{g}_{\mathrm{n}}
$$

and the left-hand side of equation (17) will be denoted as measured acceleration:

$$
\vec{a}_{\text {meas }}=\left[\mathbf{D S}^{-1}-\mathbf{C}\right] \vec{o} .
$$

Note that equation (18) does not contain any simulated data. It consists only of parameters for which realistic assumptions have been made when the simulated data have been generated. These will be unknown when LISA Pathfinder is producing real data, but the theoretical assumptions made for the data generation are used here for predicting the test mass acceleration. Thus $\vec{a}_{\text {pred }}$ is computed by applying all information about the noise sources to the known dynamical model.

The result of equation (19) gives $\vec{a}_{\text {meas }}$. All variables in this equation will initially be known:

- the dynamics $\mathbf{D}$ from analysing the model,

- the IFO cross coupling $\mathbf{S}$ from simulations,

- the controllers $\mathbf{C}$ by definition and

- the IFO output $\vec{o}$ from the measurement.

The dynamics and the interferometer cross coupling can be adapted according to the results of the on-orbit experiments. Thereby, the final result of analysing the mock interferometer data is $\vec{a}_{\text {meas }}$. 


\section{Model validation and computation of references}

In contrast to future MDCs, in MDC1 the spectrum of every noise source entering the dynamical system of LTP is known [3, 6]. As a consequence, it is possible to verify the model derived from the equations of motion by predicting the spectral content of the interferometer data:

$$
\vec{o}=\left[\mathbf{D S}^{-1}-\mathbf{C}\right]^{-1}\left[\mathbf{D S}^{-1} \vec{o}_{\mathrm{n}}+\vec{g}_{\mathrm{n}}\right] .
$$

The first term of equation (20) can be expressed as

$$
\left[\mathbf{D S}^{-\mathbf{1}}+\mathbf{C}\right]^{-1}=\left[\begin{array}{cc}
\frac{1}{\left(s^{2}+\omega_{1}^{2}\right)+C_{\mathrm{df}}} & 0 \\
\frac{-\beta}{\left(\left(s^{2}+\omega_{1}^{2}\right)+C_{\mathrm{df}}\right)\left(\left(s^{2}+\omega_{3}^{2}\right)+C_{\mathrm{sus}}\right)} & \frac{1}{\left(s^{2}+\omega_{3}^{2}\right)+C_{\mathrm{sus}}}
\end{array}\right],
$$

and the second term of equation (20) can be expressed as

$$
\mathbf{D S}^{-1} \vec{o}_{\mathrm{n}}+\vec{g}_{\mathrm{n}}=\left[\begin{array}{c}
\left(s^{2}+\omega_{1}^{2}\right) o_{\mathrm{n}_{1}}+A_{1}-A_{\mathrm{N}} \\
\beta o_{\mathrm{n}_{1}}+\left(s^{2}+\omega_{3}^{2}\right) o_{\mathrm{n}_{\Delta}}+A_{2}-A_{1}
\end{array}\right],
$$

with $\beta=\omega_{\Delta}^{2}-\delta\left(s^{2}+\omega_{3}^{2}\right)$.

Introducing the closed-loop transfer functions,

$$
S_{\mathrm{df}}=\frac{\left(s^{2}+\omega_{1}^{2}\right)}{\left(s^{2}+\omega_{1}^{2}\right)+C_{\mathrm{df}}} \quad \text { and } \quad S_{\mathrm{sus}}=\frac{\left(s^{2}+\omega_{3}^{2}\right)}{\left(s^{2}+\omega_{3}^{2}\right)+C_{\mathrm{sus}}},
$$

and solving equation (20) lead to the following expressions for $\vec{o}$ :

$$
\begin{aligned}
& o_{1}=S_{\mathrm{df}} o_{\mathrm{n}_{1}}+\frac{S_{\mathrm{df}}}{\left(s^{2}+\omega_{1}^{2}\right)}\left(A_{1}-A_{\mathrm{N}}\right) \\
& o_{\Delta}=o_{\mathrm{n}_{1}}\left[\beta \frac{S_{\mathrm{sus}}}{\left(s^{2}+\omega_{3}^{2}\right)}\left(1-S_{\mathrm{df}}\right)\right]+o_{\mathrm{n}_{\Delta}} S_{\mathrm{sus}}+A_{2} \frac{S_{\mathrm{sus}}}{\left(s^{2}+\omega_{3}^{2}\right)} \\
& -A_{1}\left[\frac{S_{\mathrm{sus}}}{\left(s^{2}+\omega_{3}^{2}\right)}\left(\beta \frac{S_{\mathrm{df}}}{\left(s^{2}+\omega_{1}^{2}\right)}+1\right)\right]+A_{N}\left(\beta \frac{S_{\mathrm{df}} S_{\mathrm{sus}}}{\left(s^{2}+\omega_{1}^{2}\right)\left(s^{2}+\omega_{3}^{2}\right)}\right) .
\end{aligned}
$$

To verify that the results of the prediction of $\vec{o}$ are the same as the simulated mock data, 'power spectral densities' (PSD) of both will be compared. The PSDs of $o_{1}$ and $o_{\Delta}$ are defined as

$$
\begin{aligned}
& \operatorname{PSD}\left(o_{1}\right)=\left\langle o_{1} \cdot o_{1}^{*}\right\rangle \\
& \operatorname{PSD}\left(o_{\Delta}\right)=\left\langle o_{\Delta} \cdot o_{\Delta}^{*}\right\rangle .
\end{aligned}
$$

From this the 'amplitude spectral density' (ASD) can be calculated:

$$
\mathrm{ASD}=\sqrt{\mathrm{PSD}}
$$

Furthermore, the spectra of the force noises $\left(A_{2}-A_{1}, A_{\mathrm{N}}, o_{\mathrm{n}}\right)$ are also given as combinations of underlying ASDs from the data generation team which makes it impossible to recover all information about the variable itself, because the phase information is missing. Then,

$\operatorname{PSD}\left(o_{1}\right)=\left|S_{\mathrm{df}}\right|^{2}\left|o_{\mathrm{n}_{1}}\right|^{2}+\frac{\left|S_{\mathrm{df}}\right|^{2}}{\left|\left(s^{2}+\omega_{1}^{2}\right)\right|^{2}}\left(\left|A_{1}\right|^{2}+\left|A_{\mathrm{N}}\right|^{2}\right)$, 


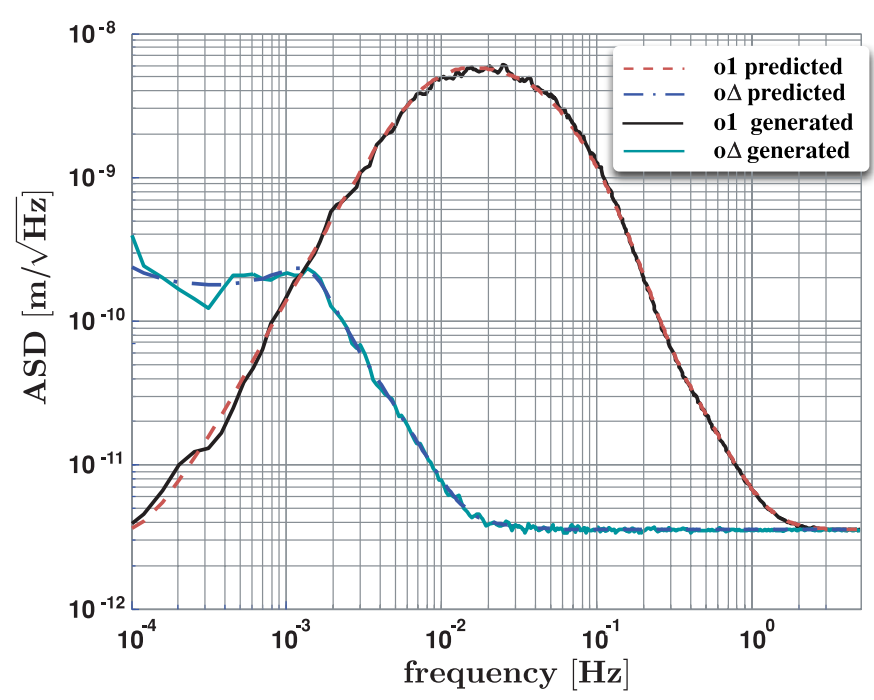

Figure 2. Comparison of the spectra of the generated and the predicted interferometer data.

$$
\begin{aligned}
\operatorname{PSD}\left(o_{\Delta}\right)=\left|o_{\mathrm{n}_{1}}\right|^{2}\left[|\beta|^{2} \frac{\left|S_{\mathrm{sus}}\right|^{2}}{\left|\left(s^{2}+\omega_{1}^{2}\right)\right|^{2}}\left(1+\left|S_{\mathrm{df}}\right|^{2}-2 \operatorname{Re}\left\{S_{\mathrm{df}}\right\}\right)\right] \\
+\left|o_{\mathrm{n}_{\Delta}}\right|^{2}\left|S_{\mathrm{sus}}\right|^{2}+\left|A_{2}\right|^{2} \frac{\left|S_{\mathrm{sus}}\right|^{2}}{\left|\left(s^{2}+\omega_{3}^{2}\right)\right|^{2}} \\
+\left|A_{1}\right|^{2} \frac{\left|S_{\mathrm{sus}}\right|^{2}}{\left|\left(s^{2}+\omega_{3}^{2}\right)\right|^{2}}\left(|\beta|^{2} \frac{\left|S_{\mathrm{df}}\right|^{2}}{\left|\left(s^{2}+\omega_{1}^{2}\right)\right|^{2}}+1+2 \operatorname{Re}\left\{\beta \frac{S_{\mathrm{df}}}{\left(s^{2}+\omega_{1}^{2}\right)}\right\}\right) \\
+\left|A_{\mathrm{N}}\right|^{2} \frac{|\beta|^{2}\left|S_{\mathrm{df}}\right|^{2}\left|S_{\mathrm{sus}}\right|^{2}}{\left|\left(s^{2}+\omega_{1}^{2}\right)\right|^{2}\left|\left(s^{2}+\omega_{3}^{2}\right)\right|^{2}} .
\end{aligned}
$$

Equation (30) describes the generation of the output data of the X1 interferometer as follows. First the transfer function of the dynamics is applied to the sum of the force noises on TM1 $\left(A_{1}\right)$ and the force noise on the spacecraft $\left(A_{\mathrm{N}}\right)$. The resulting signal gets added to the measurement noise of the X1 interferometer $o_{\mathrm{n}_{1}}$. After that the transfer function of the closedloop drag-free controller is applied to the signal, which results in the power spectral density of $o_{1}$. Predicting $o_{\Delta}$ is more complicated, because here both, $o_{1}$ and $o_{\Delta}$ couple into the result. Therefore, it is not discussed in detail but only the result is given in equation (31) and shown in figure 2. The spectra of the predicted interferometer outputs, $o_{1}$ and $o_{\Delta}$, are plotted together with those of the generated mock data that are to be analysed for comparison. These results prove the consistency of the model of the LTP dynamics with the model that was originally used for the data generation. For both interferometer outputs the curves for the predicted and the generated results agree very well. However, there are some deviations for frequencies in the sub-milihertz range, which most likely arise from averaging only very few points for the spectral estimation.

Since it has now been demonstrated that the formalism used to analyse the model is correct, the next step is to compute the expected results from the data analysis. This will provide the reference for the results obtained from calibrating the generated mock data back to acceleration. These references are given by the right-hand side of equation (17): 


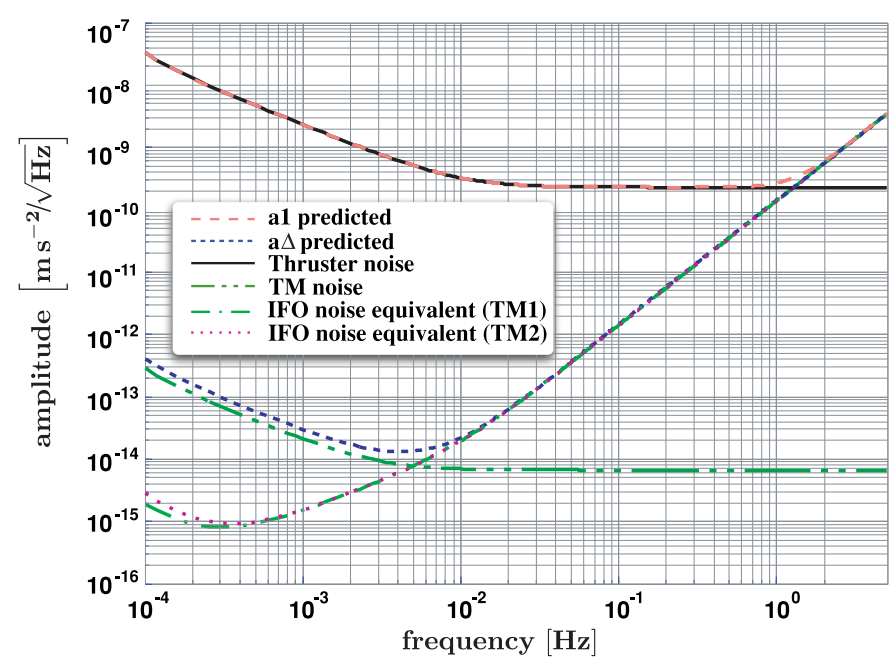

Figure 3. The impact of the different force noises on the predicted accelerations of TM1 and the differential acceleration of both TMs.

$$
\vec{a}=\mathbf{D} \vec{o}_{\mathrm{n}} \mathbf{S}^{-1}+\vec{g}_{\mathrm{n}} .
$$

The PSD of $\vec{a}$ is defined as

$$
\begin{aligned}
& \operatorname{PSD}\left(a_{1}\right)=\left|\left(s^{2}+\omega_{1}^{2}\right)\right|^{2}\left|o_{\mathrm{n}_{1}}\right|^{2}+\left|A_{1}\right|^{2}+\left|A_{\mathrm{N}}\right|^{2}, \\
& \operatorname{PSD}\left(a_{\Delta}\right)=|\beta|^{2}\left|o_{\mathrm{n}_{1}}\right|^{2}+\left|\left(s^{2}+\omega_{3}^{2}\right)\right|^{2}\left|o_{\mathrm{n}_{\Delta}}\right|^{2}+\left|A_{2}\right|^{2}+\left|A_{1}\right|^{2} .
\end{aligned}
$$

Figure 3 shows the predictions for the accelerations of test mass $1\left(a_{1}\right)$ and the differential acceleration between the test masses $\left(a_{\Delta}\right)$, respectively. The spectra of the noise sources described earlier in this document are plotted for comparison. One can see that $\tilde{a}_{1}$ is dominated by the thruster noise $A_{\mathrm{N}}$ up to a frequency of about $1 \mathrm{~Hz}$. This means that at these frequencies one cannot distinguish between test mass movement and spacecraft movement, which is consistent with our model because the spacecraft follows the motion of TM1 by applying a force via the micro-Newton thrusters. Force noise acting on the thrusters should directly couple into the measurement of the acceleration of TM1. At higher frequencies the interferometer noise dominates the measurement. For frequencies up to $10 \mu \mathrm{Hz}$, the differential test mass acceleration $\tilde{a}_{\Delta}$ is dominated by the test mass noise and for higher frequencies the interferometer measurement dominates the result.

\section{Data analysis}

The aim of the analysis of the simulated data is to extract an estimate of the differential acceleration of the two test masses. This is done by applying the same dynamical model to the data that has been used to generate it. In order to analyse the data, in addition to the system's dynamics only the transfer functions of the controllers and their delays must be known. The analysis is done according to the left-hand side of equation (17):

$$
\vec{a}=\left[\mathbf{D S}^{-\mathbf{1}}+\mathbf{C}\right] \vec{o},
$$




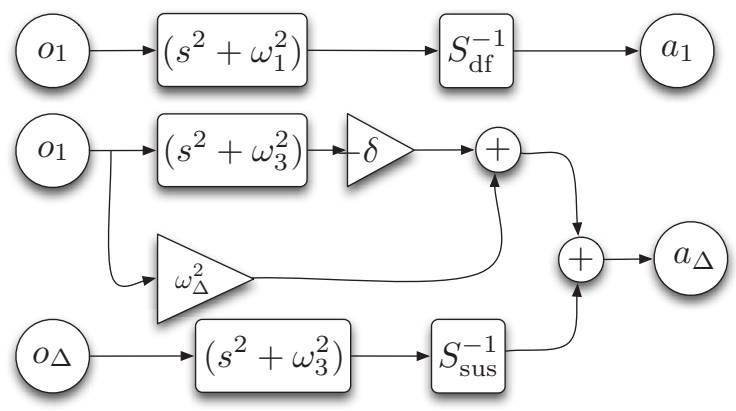

Figure 4. Data analysis of IFO output data. In the case of analysis in the Fourier domain, the boxes represent transfer functions, and in the case of time-domain analysis corresponding filters are defined.
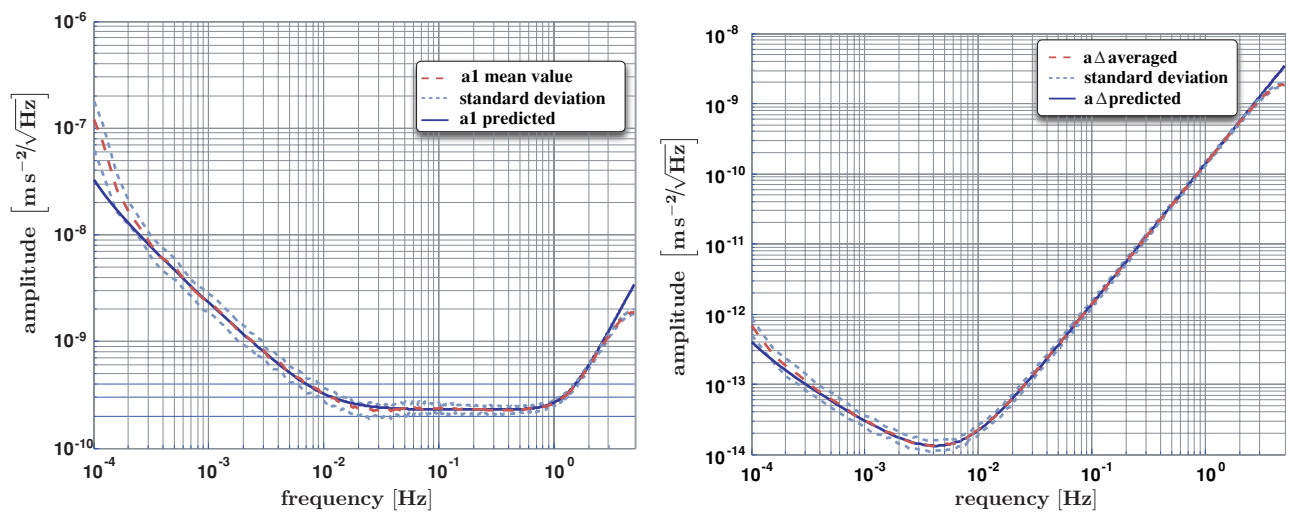

Figure 5. Result of data analysis: mean of calibration to $a_{1}$ (left) and $a_{\Delta}$ (right) for 11 data sets. Dashed lines correspond to their standard deviation.

stating that differentiating the interferometer output twice and correcting for the coupling as well as for commanded forces gives the estimates of the TM accelerations:

$$
\left[\begin{array}{c}
\mathrm{a}_{1} \\
\mathrm{a}_{\Delta}
\end{array}\right]=\left[\begin{array}{cc}
\left(s^{2}+\omega_{1}^{2}\right) S_{\mathrm{df}}^{-1} & 0 \\
\omega_{\Delta}^{2}+\left(s^{2}+\omega_{3}^{2}\right) \cdot(-\delta) & \left(s^{2}+\omega_{3}^{2}\right) S_{\text {sus }}^{-1}
\end{array}\right]\left[\begin{array}{c}
\mathrm{o}_{1} \\
\mathrm{o}_{\Delta}
\end{array}\right] .
$$

The procedure is also shown in figure 4. The output of the X12 interferometer couples into the calibration of the differential test mass acceleration, which is the reason for the pipeline of $a_{\Delta}$ being more complicated than that of $a_{1}$.

The data analysis of the simulated interferometer data can be done in the Fourier domain as well as in the time domain. In the case of the analysis in the time domain, the elements in figure 4 are generated using filters. Figure 5 shows the test mass accelerations which have been found by analysing 11 sets of simulated interferometer data and averaging the results. The error curves correspond to the standard deviation of the displayed mean and are indicated as dashed lines. The solid line represents the theoretical curve computed above using equation (34). It can be seen that the results of the data analysis agrees very well with the theoretical predictions derived from analysing the dynamical model of LTP. However, there are some small deviations: 
- At low frequencies these arise from the estimation of the spectral densities.

- The discrepancy between reference and analysis above $1 \mathrm{~Hz}$ arises from the method of numerical differentiation used in the time-domain analysis.

- The very small deviations around $10 \mathrm{mHz}$ for $a_{1}$ arise from slightly different controller transfer functions for the analysis and for the generation of the data. For the ongoing investigations of MDC2 these have been adapted.

Analysing the data in the Fourier domain means that all elements in figure 4 are transfer functions. Such that initially the PSD of $\vec{o}$ is estimated and after that the transfer functions are applied. From equation (36) and figure 4, the following equations follow for the result of the data analysis in the frequency domain:

$$
\begin{aligned}
& \operatorname{PSD}\left(a_{1}\right)=\left|o_{1}\right|^{2} \frac{\left|\left(s^{2}+\omega_{1}^{2}\right)\right|^{2}}{\left|S_{\mathrm{df}}\right|^{2}}, \\
& \operatorname{PSD}\left(a_{\Delta}\right)=\left|o_{1}\right|^{2}|\beta|^{2}+\left|o_{\Delta}\right|^{2} \frac{\left|\left(s^{2}+\omega_{3}^{2}\right)\right|^{2}}{\left|S_{\text {sus }}\right|^{2}}+\beta \frac{\left(s^{2}+\omega_{3}^{2}\right)^{*}}{S_{\text {sus }}^{*}}-o_{1, \Delta}+\beta^{*} \frac{S \omega_{3}}{S_{\text {sus }}} o_{1, \Delta},
\end{aligned}
$$

where $o_{1, \Delta}=\left\langle o_{1} o_{\Delta}^{*}\right\rangle$. The resulting functions for $\vec{a}$ in the frequency domain are not plotted here, since they agree with the results of the time-domain analysis (figure 5), apart from the deviations above $1 \mathrm{~Hz}$ which arise from the differentiation method used that is described in [7]. From the results shown it can be said that the theoretical predictions are in very good agreement with the outcome of the data analysis.

\section{Conclusion}

The aim of this work was to analyse mock interferometer data that have been simulated using a simplified model of the LTP dynamics. For doing this the dynamical model had to be verified first. This was done by finding an analytical expression for the interferometer data by applying the derived equations of motion of the two test masses and then comparing the results with the data received from the data generation group. The spectra of the analytical functions, the socalled predictions, matched very well the spectra obtained from the simulated interferometer data (see figure 2). This means that the two dynamical models derived independently from each other by two different groups using different software tools result in the same power spectra of the interferometer output data. Due to this result, the derived dynamical model is deemed to be verified (see section 3). Only from a verified dynamical model of LTP it made sense to calibrate the interferometer data to test mass acceleration, not using any information about noises acting on the system just like it would be during the mission. Section 4 shows that the resulting power spectra of the test mass accelerations match those predicted from the noises acting on the system in section 3 (compare figure 5). To conclude, the accelerations of the two test masses moving along the axis connecting them were derived successfully from simulated interferometer output data independent of the knowledge of any noise sources.

For calibrating the interferometer output data to test mass accelerations, analysis tools and procedures for the LISA Pathfinder mission were developed.

Although the model used for MDC1 is simplified, it could be sufficient for the analysis of an optimized experiment, where the cross-talks from other axes are minimized. Another important aim achieved was to find a common language between the groups analysing and generating the mock interferometer data. By overcoming this first obstacle of completely understanding the LTP dynamics and all its contributions such as controllers and force noises, a lot of experience has been gained. 
The work on future MDCs will profit a lot from the experiences made in MDC1. In MDC2 the analysis will be focused on parameter estimation: values of controller gains, stiffness coefficients and interferometer cross-talk will not be communicated but have to be estimated, e.g., by applying appropriate fitting routines. Future MDCs will then be based on models which will have a stepwise increase in complexity. MDC3, for example, will probably include cross-talk from other axes.

\section{Acknowledgments}

We gratefully acknowledge support by Deutsches Zentrum für Luft- und Raumfahrt (DLR) (references 50 OQ 0601).

\section{References}

[1] Anza S et al 2005 The LTP experiment on the LISA Pathfinder mission Class. Quantum Grav. 22 125-38

[2] Steier F et al 2009 The end-to-end testbed of the optical metrology system on-board LISA Pathfinder Class. Quantum Grav. 26094010

[3] Vitale S 2007 Method of generation and accompanying notes for the first set of mock data for the LTP data analysis Technical Report S2-UTN-TN-3058 Issue/Rev. 1.1, University of Trento

[4] Hewitson M et al 2009 Data analysis for the Lisa Technology Package Class. Quantum Grav. 26094003

[5] Fichter W, Gath P, Vitale S and Bortoluzzi D 2005 The LTP experiment on the LISA Pathfinder mission Class. Quantum Grav. 22 139-48

[6] Vitale S 2007 Technical Report S2-UTN-TN-3060 Issue/Rev. 1.0, University of Trento

[7] Ferraioli L et al 2009 Derivative estimation for assessing low frequency noise in LISA Pathfinder data reduction Class. Quantum Grav. 26094013 